\title{
Multivariate Curve Resolution to Determine Fully-Embedded-Particle Compositions in STEM-EDS Spectrum Images
}

\author{
Chad M. Parish \\ Materials Science and Technology Division, Oak Ridge National Laboratory, Oak Ridge, TN 37831
}

Fine-scale precipitates and core/shell-structured nanoparticles are difficult to analyze. For example, radiation-induced particles or oxide dispersions in structural materials can often be only a few nanometers in diameter. Small particles will be embedded fully within the thickness of all but the thinnest foils, and compositional analyses of the particles using energy dispersive spectroscopy (EDS) or electron energy loss spectroscopy (EELS) will be compromised by the matrix contribution. Here, it is hypothesized that by applying multivariate curve resolution (MCR) methods as described in the chemometrics literature to STEM-EDS spectrum images (SIs), the embedded particles' spectra can be separated from the surrounding matrix spectrum.

Previous work on "NYTO" $\mathrm{TiO}_{2} / \mathrm{Y}_{2} \mathrm{O}_{3}$ oxide dispersion-strengthened Ni showed fine particles embedded in a thin-foil matrix [1]. Principal component analysis (PCA) of an experimental SI was used to create a phase map, and DTSA-II software [2] was used to simulate $\mathrm{Ni}, \mathrm{TiO}_{2}$, and $\mathrm{Y}_{2} \mathrm{O}_{3}$ EDS spectra. From these phase maps and EDS spectra, a 50×50 -pixel STEM-EDS spectrum image was simulated of $\mathrm{TiO}_{2}$ and $\mathrm{Y}_{2} \mathrm{O}_{3}$ particles fully embedded in a $\mathrm{Ni}$ thin-foil with an average signal level of 1,000 X-ray counts / pixel. Simulations, rather than experimental data, were used so that the correct particle spectral responses were exactly known for comparison to the resolved spectra. Using only the information in the SI, such as via PCA, it is impossible a priori to uniquely determine the spectra for fully embedded particles [3]; a rotational ambiguity remains which cannot be resolved. However, if additional information is used to constrain the bilinear model, the bands of rotational ambiguity can be significantly reduced or possibly even collapsed to determine a unique solution. Freely available software [4] was used in conjunction with local-rank constraints [5] and a closure constraint on the scores to perform MCR on the simulated NYTO spectrum image. Fig. 1 shows the MCR score images (left), and the MCR loading spectra and difference between the loadings and known starting spectra (right). The $\mathrm{Y}_{2} \mathrm{O}_{3}$ and $\mathrm{TiO}_{2}$ spectra are nearly fully resolved; only slight contamination by Ni-K and -L peaks remain in the oxide spectra, which indicate that the embedded particle compositions were nearly resolved by constrained MCR.

Fig. 2 shows the same techniques applied to the experimental data. Some Ni K/L contribution is observed in the $\mathrm{TiO}_{2}$-rich loading, and a larger $\mathrm{Ni} \mathrm{K} / \mathrm{L}$ contribution (along with a small $\mathrm{Ti} \mathrm{K} / \mathrm{L}$ contribution) is seen in the $\mathrm{Y}_{2} \mathrm{O}_{3}$-rich loading. The "true" loadings are unknown, but phase diagrams indicate only $\sim 2 \% \mathrm{NiO}$ solubility in $\mathrm{TiO}_{2}$, negligible $\mathrm{NiO}$ solubility in $\mathrm{Y}_{2} \mathrm{O}_{3}$, and two Ti-Y-O mixed oxides $\left(\mathrm{Ti}_{2} \mathrm{Y}_{2} \mathrm{O}_{7}\right.$ and $\left.\mathrm{TiY}_{2} \mathrm{O}_{5}\right)$. Simple elemental maps extracted from the SI indicate low levels of $\mathrm{Ti}$ in the small Y-rich particles [1]. The greater confusion in the experimental data is likely due to uncertainties in local rank computations and variations in foil thickness confusing the score closure constraints. Further technique development is underway. [6]

[1] J. Bentley \& D. T. Hoelzer, Micros. Microanal., 15(Suppl. 2) (2009) 1368.

[2] DTSA-II software: http://www.cstl.nist.gov/div837/837.02/epq/dtsa2/index.html

[3] R. Manne, Chemom. Int. Lab. Sys., 27 (1995) 89.

[4] J. Jaumot et al., Chemom. Int. Lab. Sys., 76 (2005) 101; http://www.ub.edu/mcr/welcome.html

[5] A. de Jaun et al., J. Chemometrics, 22 (2008) 291.

[6] Research supported by the Shared Research Equipment (SHaRE) User Program, sponsored at Oak Ridge National Laboratory by the Scientific User Facilities Division, Office of Basic Energy Sciences, U.S. Department of Energy and by ORNL's Laboratory Directed Research and Development Weinberg Fellowship Program. Data provided by Drs. J. Bentley and D. T. Hoelzer. 

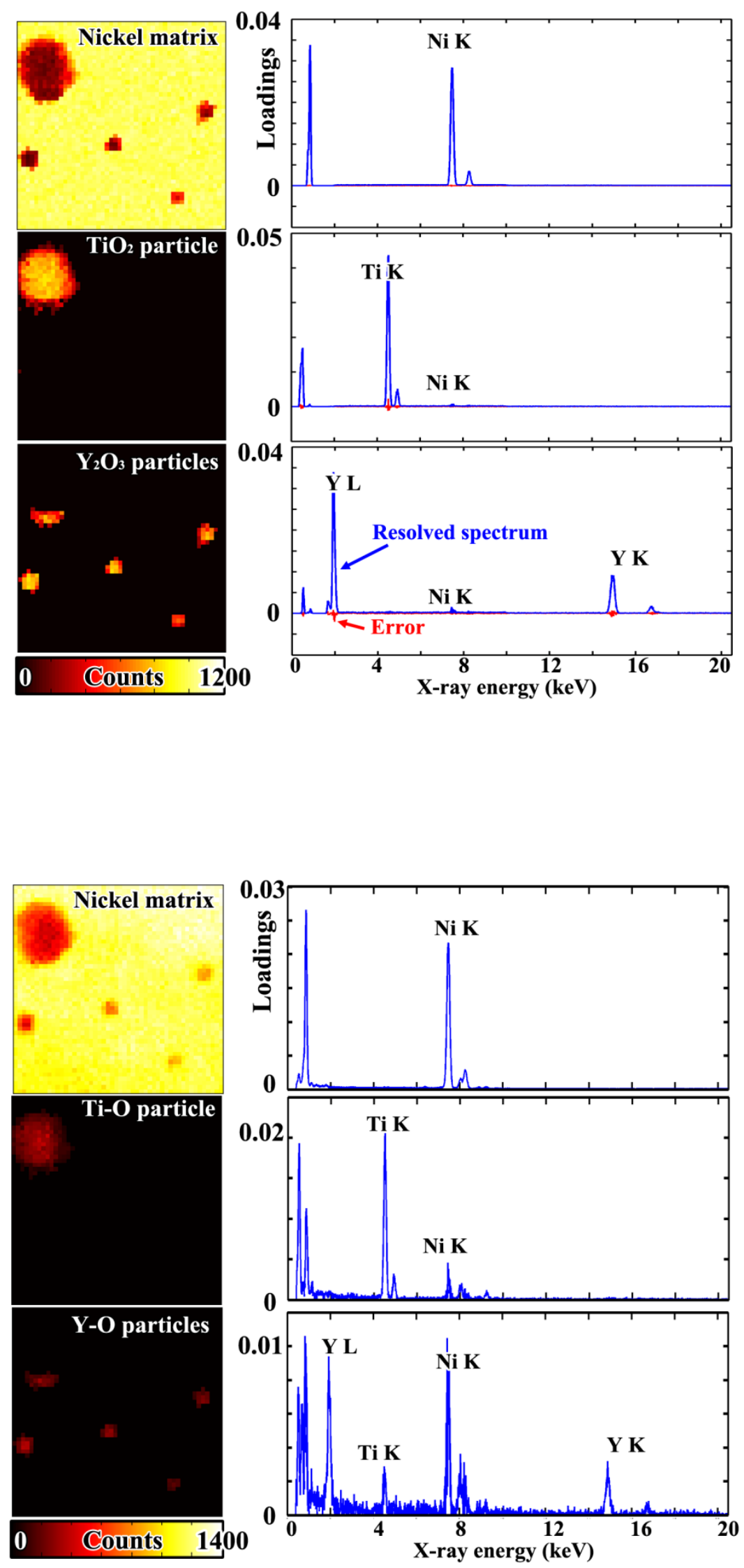

Figure 1: Left column shows score images and right column shows resolved loading spectra for Ni matrix, $\mathrm{TiO}_{2}$ particle, and $\mathrm{Y}_{2} \mathrm{O}_{3}$ particles in the simulated spectrum image. Blue curves are resolved spectra and red curves the error compared to known simulation starting point. Image sizes: $50 \times 50$ pixel simulation. Loading spectra integrated areas $=1.0$.

Figure 2: Left column shows score images and right column shows resolved loading spectra for $\mathrm{Ni}$ matrix, $\mathrm{TiO}_{2}$-rich particle, and $\mathrm{Y}_{2} \mathrm{O}_{3}$-rich particles in the experimental spectrum image. Blue curves are resolved spectra. Image sizes: $100 \times 100 \mathrm{~nm}$, $50 \times 50$ pixel scan.

Loading spectra integrated areas $=1.0$. Data from [1]. 